\title{
Editorial
}

Pathologe 2013 · 34:292

DOI 10.1007/s00292-013-1779-9

Online publiziert: 10. Juli 2013

(c) Springer-Verlag Berlin Heidelberg 2013

\section{Röcken ${ }^{1} \cdot$ T. Rüdiger ${ }^{2} \cdot$ B. Koch ${ }^{3}$}

${ }^{1}$ Institut für Pathologie, Christian-Albrechts-Universität Kiel

2 Pathologisches Institut, Städtische Kliniken Karlsruhe gGmbH, Karlsruhe

${ }^{2}$ Springer-Verlag, Heidelberg

\section{Zertifizierte Fortbildung in Der Pathologe}

Sehr geehrte Leserinnen und Leser,

im Beschlussprotokoll des 106. Deutschen Ärztetages vom 20. bis 23.05.2003 in Köln findet sich folgende Passage:

„Die Definition guter medizinischer Versorgung kann allerdings nur dann in gutes ärztliches Handeln münden, wenn der Transfer zum Arzt gewährleistet ist. Dazu sind kontinuierliche berufsbegleitende Fortbildung („,continuing medical education", CME) sowie kontinuierliche berufliche Kompetenzerhaltung und -entwicklung (, ,continuing professional development", $C P D$ ) erforderlich" (www.bundesaerztekammer.de, Zugriff am 05.04.2013).

Wie Sie wissen, ist seit 2005 der Nachweis ärztlicher Fortbildung für alle Ärzte, ob niedergelassen oder im Krankenhaus tätig, zur gesetzlich verankerten Pflicht geworden.

\section{) Nutzen Sie die Möglichkeit, mit Der Pathologe CME-Punkte zu sammeln!}

Als Organ der Deutschen Gesellschaft für Pathologie, der Deutschen Abteilung der Internationalen Akademie für Pathologie, der Österreichischen Gesellschaft für Pathologie, der Schweizerischen Gesellschaft für Pathologie und des Bundesverbandes Deutscher Pathologen bietet deshalb auch Der Pathologe ab dieser Ausgabe eine aktuelle und umfassende Fortbildung mit der Möglichkeit, CME-Punkte zu sammeln. Zusammengestellt und koordiniert werden die strukturiert aufge- arbeiteten Weiterbildungsbeiträge durch Herrn Prof. Dr. Christoph Röcken, Kiel, und Herrn Prof. Dr. Thomas Rüdiger, Karlsruhe.

Seit dem Jahr 2000 bietet der SpringerVerlag in seinen deutschsprachigen medizinischen Fachzeitschriften die Möglichkeit, CME-Punkte zu sammeln. Das Fortbildungsangebot ist zertifiziert von der Nordrheinischen Akademie für Ärztliche Fort- und Weiterbildung sowie der Landesärztekammer Hessen und ist damit bundesweit anerkennungsfähig.

Durch Beantwortung der 10 Multiplechoice-Fragen, die zu jedem CME-Beitrag gehören, können pro Fortbildungseinheit 3 CME-Punkte erworben werden. Abonnenten von Der Pathologe können das Online-CME-Angebot ihrer Zeitschrift unter www.springermedizin.de/eAkademie kostenlos nutzen.

Der erste CME-Beitrag in Der Pathologe behandelt das Thema Mammakarzinom und gibt aktuelle Empfehlungen für Pathologen auf der Basis der S3-Leitlinie. Er bildet den Anfang einer Reihe von Beiträgen, die hoffentlich für alle Leser, $d$. h. Fachärzte und Fachärztinnen für Pathologie und Weiterbildungsassistenten und -assistentinnen gleichermaßen von Interesse sind. In den folgenden Ausgaben werden Themen wie Prostatapathologie, Molekularpathologie und Vaskulitiden aufgegriffen.

Die Herausgeber sind natürlich sehr an Ihrer Meinung interessiert: Kritik, Anregungen, aber sicher auch Lob, sind jederzeit willkommen.

Die Rubrikherausgeber und die Redaktion von Der Pathologe wünschen Ihnen, den Leserinnen und Lesern, eine interessante Lektüre und natürlich viel Erfolg bei der Beantwortung der CMEFragen!

Ihre

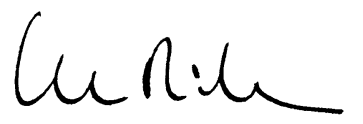

Prof. Dr. C. Röcken, CME-Herausgeber

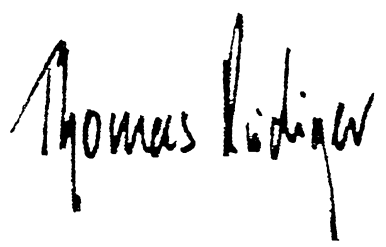

Prof. Dr. T. Rüdiger, CME-Herausgeber

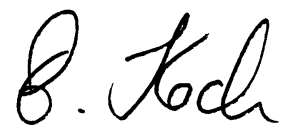

Dr. B. Koch, Redaktionsleitung Der Pathologe

\section{Korrespondenzadressen}

Prof. Dr. C. Röcken

Institut für Pathologie,

Christian-Albrechts-Universität Kiel

Arnold-Heller-Straße 3, 24105 Kiel

christoph.roecken@uksh.de

\section{Prof. Dr. T. Rüdiger}

Pathologisches Institut,

Städtische Kliniken Karlsruhe gGmbH

Moltkestr. 90, 76133 Karlsruhe

thomas.ruediger@klinikum-karlsruhe.de 